\title{
Phonological codes are early sources of constraint in visual semantic categorization
}

\author{
MIRA PETER and M. T. TURVEY \\ University of Connecticut, Storrs, Connecticut \\ and Haskins Laboratories, New Haven, Connecticut
}

\begin{abstract}
Two experiments were directed at early phonological activation in the semantic categorization task. In Experiment 1, briefly exposed targets homophonic to category exemplars (Rows for the category A FLOWER), and their graphemic controls (ROBS), were judged for category membership with and without a backward pattern mask. False positives were greater for Rows than ROBS to the same degree under both unmasked and masked conditions. In Experiment 2, false positives were examined in the semantic categorization task under backward dichoptic masking by pseudowords that were, in turn, masked monoptically by a pattern mask. Briefly exposed homophones (e.g., WEAK), masked by a phonologically similar pseudoword ("feek"), a graphemic control ("felk"), or an unrelated pseudoword ("furt"), were categorized as category exemplars (A UNIT OF TIME). The difference in false positives was significant for WEAK-feek versus WEAK-furt, but not for WEAKfelk versus WEAK-furt. It was suggested that the persistence of the homophonic effects under the pattern masking of Experiment 1 and their amplification under the phonological masking of Experiment 2 were because phonological codes cohere rapidly and provide, thereby, immediately available constraints on semantic processing.
\end{abstract}

The dual-process theory advanced by Coltheart (1978) has motivated most of the contemporary research on recognizing and pronouncing words. Two independent processes-a direct, visual process and a mediated, phonological process-are said to govern the accessing of the internal lexicon. According to the theory, these two independent processes are distinguishable in prominence and in the types of stimuli to which they most aptly apply. The primary access route is the visual process, which entails a mapping between orthographic features of individual printed/written words and lexical representations. The secondary access route is the mediated process, which involves a set of grapheme-phoneme correspondence rules turning spellings into phonological representations, and a subsequent mapping from these phonological representations onto lexical entries. For the fluent reader, familiar words and words with exceptional spellings are read primarily through the faster, direct route; the slower phonological mediation route is prominent in the reading of new words and nonwords.

Since the formulation of dual-process theory, arguments have been marshalled that the appeal to a rule-governed phonological process to access the lexicon is superfluous (Humphreys \& Evett, 1985): Lexical access is achieved for all word forms in a word-specific manner, by the direct visual route. The latter conclusion, however, is contradicted by a wide range of studies with Serbo-Croatian

\footnotetext{
This research was supported by NICHD Grant HD-01994 awarded to the Haskins Laboratories. We acknowledge Arthur Abramson's help in constructing the homophonic and graphemic pseudowords. Correspondence concerning this article should be addressed to M. Peter or M. T. Turvey, Haskins Laboratories, 270 Crown Street, New Haven, CT 06511.
}

language materials (summarized in Lukatela \& Turvey, $1990 \mathrm{~b}, 1991$ ) and new classes of evidence from recent studies with English language materials (Lukatela \& Turvey, 1991, 1993; Perfetti \& Bell, 1991; Perfetti, Bell, \& Delaney, 1988; Van Orden, 1987; Van Orden, Johnston, \& Hale, 1988). Collectively, the outcomes of these studies suggest that the primary constraint on lexical access is phonological, not visual. It has been proposed that this phonological constraint is not exerted by a mechanism of explicit symbol-manipulating rules, as proposed in classical dual-process theory, but by a mechanism incorporating the continuous statistical regularity relating orthographic and phonological patterns (Van Orden, Pennington, \& Stone, 1990).

The research reported in the present article builds on two of the recent lines of support for phonological mediation in English, specifically, research on semantic categorization of homophones (Van Orden, 1987; Van Orden et al., 1988) and on identification of briefly exposed letters under homophonic backward masking (Perfetti \& Bell, 1991; Perfetti et al., 1988). In Van Orden's experiments, targets that were either homophonic or graphemically similar to category examples (ROws and ROBS for the category A FLOWER) were judged as category exemplars. False-positive classifications were found to be significantly higher for homophonic category examples (ROws) than for their respective controls (ROBS). Additionally, false-positive classifications of homophonic pseudoword foils (e.g., ROAs) matched those of homophonic words. The reading given to these results on semantic categorization was that phonological activation occurs automatically in lexical access. Apparently, a visually presented letter string activates a set of candidates that share the phono- 
logical representation with the target (Rows), including the category example (ROSE), thus producing higher error rates on homophonic targets than on their spelling controls (Van Orden, 1987; Van Orden et al., 1988).

In Perfetti's experiments, a briefly presented target word in lowercase (e.g., "fame") was immediately followed by either a phonologically similar pseudoword in uppercase (e.g., PHAIM), a graphemically similar pseudoword in uppercase (e.g., PHAFT), or an unrelated, control pseudoword in uppercase (e.g., CRON). The pseudoword mask itself was immediately followed by a pattern mask to reduce its identification and, thereby, guessing strategies about target/pseudoword mask relations (Naish, 1980). It was assumed that the disruptive effect of backward visual masking on the target would be reduced if the mask was either graphemically or phonologically similar to the target word. The results revealed that graphemic overlap between the target and the mask reduced the masking effect, and that phonological overlap reduced the masking effect even further (Perfetti \& Bell, 1991; Perfetti et al., 1988)-a pattern of results that was observed over variable combinations of target exposure and mask duration. Apparently, phonological activation occurs before the target is identified and is influenced by the phonological properties of the mask (see also Lukatela \& Turvey, 1990a). The results comport with the assumption that the activation of phonological information during the identification of printed English words is both a general and early aspect of the identification process.

With respect to adjudicating on the issue of phonological mediation, the significance of the semantic categorization task is that word meanings must be accessed in order to satisfy the task requirements. This is in contrast to what is needed minimally, presumably, to satisfy the requirements of the commonly used tasks of lexical decision and rapid naming. Consequently, to demonstrate phonological effects in the semantic categorization task is to strengthen the hypothesis that phonological mediation underlies ordinary word recognition (Van Orden, 1987). Our goal in the present research was to determine whether the phonological coding manifest in the semantic categorization task is both routine and early. To this end, Experiment 1 was directed at examining the production of false positives with and without time constraints (as manipulated through backward pattern masking), and Experiment 2 was directed at examining the production of false positives under conditions of masking by phonologically similar stimuli, that is, in a paradigm that embeds the Van Orden procedure within the Perfetti procedure.

\section{EXPERIMENT 1}

As noted in a recent survey by Van Orden et al. (1990), an important hypothesis of many word-recognition models is that phonological codes are late sources of constraint on recognition relative to the source of constraint provided by direct visual access (e.g., Coltheart, Davelaar, Jonasson, \& Besner, 1977; McCusker, Hillinger, \& Bias, 1981; Seidenberg, 1985). If this hypothesis is true, then pre- sentation conditions that force reliance upon the earliest available codes should be conditions in which phonological effects are reduced or absent. Presenting a patterned mask after a to-be-categorized word seems to be a condition of presentation that compresses the time available for processing (Michaels \& Turvey, 1979; Turvey, 1973). Consequently, with respect to the semantic categorization task, false positives to brief exposures of ROWS and ROBS (as "exemplars" of the category A FLOWER) should be more nearly equal when they are immediately followed by a backward pattern mask than when they are not. Stated more specifically, the delayed-phonology hypothesis of dual-process theory predicts an interaction between the contrast of Rows versus RoBS and the contrast between unmasked versus masked, with a smaller difference in false positives under the masked condition. In comparison, the claim that phonological coding occurs very early in the course of word recognition leads to the expectation that the contrast between Rows and RoBs should persist under masking; the relation between target type and presentation condition is likely to be either an additive relation or an interactive relation in which the Rows versus ROBS difference is larger under the masked condition. Van Orden (1987) found more false positives to Rows than to ROBS in an experiment (Experiment 2) in which the stimulus onset asynchrony between target and mask was close to the threshold of each individual subject. The Rows versus ROBS difference in that experiment compared favorably to the difference observed in Van Orden's (1987) Experiment 1, in which the stimulus onset asynchrony was $500 \mathrm{msec}$. In the present experiment, all the word targets were of the same brief duration and either were or were not immediately followed by a patterned mask. The aim of Experiment 1, therefore, was to confirm the homophonic effect in semantic categorization and to provide a direct test of the possibility that the Rows versus ROBS difference, found under viewing conditions that allow unhurried processing, persists to the same degree (at least) or magnifies (at most) under viewing conditions that severely restrict processing time.

\section{Method}

Subjects. Forty-four undergraduate students at the University of Connecticut participated in the experiment. All were native English speakers, and all had normal or corrected-to-normal vision. The subjects received credit for a course requirement. Each subject was randomly assigned to one of two experimental groups.

Materials. The original stimuli from Van Orden (1987) were used in the experiment. The homophonic targets and their graphemic controls are given in Appendix A. There were 18 practice trials and 80 experimental trials. All targets were legal English words. Of the experimental trials, $25 \%$ were homophonic to the category exemplar (e.g., Rows for the category A FLOWER), and $25 \%$ were their graphemic controls (e.g., ROBs). None of the targets was the category exemplar. The remaining $\mathbf{4 0}$ targets were the exemplars of the given category. The targets were printed in capital letters.

Procedure. There were two groups of subjects in the experiment, Group $\mathrm{U}$ and Group $\mathbf{M}$ (for unmasked and masked, respectively). Both groups saw the same set of targets. The only difference between the two groups was that, for Group M, the target word was immediately followed (interstimulus interval, or ISI, of $0 \mathrm{msec}$ ) by 
a pattern mask (\#\#\#\#). The general procedure was the same for both groups. The subjects sat before an Apple Ile computer and fixated their eyes at a point on the computer screen. On each trial, a subject was presented with a category name, read aloud by the experimenter, which was immediately followed by the presentation of a target word on the screen. The subject was instructed to respond "yes"' if the target word was the category exemplar and "no" if it was not. The target exposure duration was a nominal $45 \mathrm{msec}$. (Display changes occurred within the standard 16-msec scan rate of the Apple Ile monitor, meaning that all actual durations of the nominated 45-msec exposure varied in a random manner between 45 and $61 \mathrm{msec}$.) Each subject received 18 practice trials that were immediately followed by 80 experimental trials. All the subjects were presented with the same random order of both practice and experimental trials. Both speed and accuracy were stressed.

\section{Results and Discussion}

The percentages of false positives for Groups $U$ and $M$ are presented in Table 1 . A $2 \times 2$ analysis of variance (ANOVA) was conducted on the false positives with the within-subject factor of target type (ROWs vs. ROBS) and the between-subject factor of presentation condition (unmasked vs. masked). The main effect of target type (Rows $=17.56$ vs. ROBS $=6.76$ ) was significant [minimum $F^{\prime}(1,53)=15.86, p<.001$; for subjects, $F(1,42)=$ 95.64, $p<.001$; for stimuli, $F(1,38)=19.00, p<$ $.001]$. Also significant was the main effect of presentation [unmasked $=6.31 \%$ vs. masked $=18.01 \%$; minimum $F^{\prime}(1,68)=33.39, p<.001$; for subjects, $F(1,42)$ $=45.60, p<.001$; for stimuli, $F(1,38)=124.75, p<$ $.001]$. Clearly, the effect of the mask was to increase the overall number of false positives. Did it, in addition, modify the Rows versus ROBs difference? In particular, did masking reduce the difference, as expected from the delayed-phonology hypothesis? Contrary to the latter hypothesis, the interaction of target type $\times$ presentation was not significant with a suggestion-in further contradiction of the hypothesis-that masking magnified the Rows versus ROBS difference [difference in unmasked $=8 \%$ vs. difference in masked $=13 \%$, minimum $F^{\prime}(1,56)=.54$, $p>.05$; for subjects, $F(1,42)=2.713, p>.05$; for stimuli, $F(1,38)=5.28, p<.05$ ]

The results from the present experiment on semantic categorization corroborate those reported previously (Van Orden, 1987; Van Orden et al., 1988) in demonstrating a reliable tendency for Rows to be categorized as A FLOWER more often than ROBS. They extend previous research in demonstrating that the difference in false positives between Rows and ROBS is of the same magnitude under both unmasked and masked conditions. Insofar as the

Table 1

Mean Percentages and Standard Deviations of

False-Positive Responses to Homophones (e.g., Rows) and Spelling Controls (ROBS), in Experiment 1

\begin{tabular}{|c|c|c|c|c|}
\hline & \multicolumn{2}{|c|}{ Unmasked } & \multicolumn{2}{|c|}{ Masked } \\
\hline & $M$ & $S D$ & $M$ & $S D$ \\
\hline Homophones & 10 & 7.4 & 24 & 9.3 \\
\hline Spelling controls & 2 & 2.2 & 11 & 6.2 \\
\hline
\end{tabular}

backward pattern masking of the present experiment reduced the time available for processing (to $45 \mathrm{msec}$ in the present experiment), the constancy of the difference implies that the constraint on semantic categorization provided by phonological codes comes into operation very early.

The categories used in the present experiment were, with perhaps three exceptions (flowers, animals, food), of the kind that possess relatively few members. On the basis of preceding research, therefore, the observed homophonic effect should be frequency independent (Jared \& Seidenberg, 1991; Van Orden, 1987). The stimuli ranged in frequency from 0 to 1,125 with a mean of 77 (Kučera \& Francis, 1967). There were no correlations (negative or otherwise) between frequency and the size of the homophonic effect for the masked or unmasked conditions ( $r=.003$ and $r=.002$, respectively). Thus, the results suggest that for the present narrow categories, early phonological activation contributes to the activation of word meaning over a wide range of frequencies.

\section{EXPERIMENT 2}

As noted, Perfetti and his colleagues (Perfetti \& Bell, 1991; Perfetti et al., 1988; Perfetti, Zang, \& Berent, 1992) investigated the effects of the early activation of phonological information by using word identification under backward masking. This task requires a "shallow" level of processing; namely, it does not necessarily involve semantic access. To examine whether the observed activation of early phonological codes could be generalized beyond the specific characteristics of the word identification/backward masking task, we combined the Perfetti task with the semantic categorization task. Other possible tasks that could be yoked to Perfetti's are naming and lexical decision. Arguments have been raised, however, about their capacity to address the nature of the representations that activate a word's meaning. In contrast, Van Orden's semantic categorization requires, by definition, activation of a word's meaning, and as such it permits the exploration of factors that influence semantic processes. Thus, a combination of Van Orden's and Perfetti's tasks should enable us to (1) further explore the issue of the influence of phonological codes on accessing word meaning, and (2) strengthen the argument that these codes are computed prior to complete word identification.

When a briefly exposed target word is closely followed by an overlapping pattern, the processing of the target word can be interrupted so that the information derived from it is incomplete (Michaels \& Turvey, 1979; Turvey, 1973). If under certain stimulus parameters the target word was rarely identified, then it might be argued that, under those parameters, the internal lexicon was rarely accessed. That is, the mask's effect was such that it impeded the acquisition of information adequate enough to access the target's lexical entry. Now suppose that a mask with the same physical parameters and figural complexity as the original, but related to the target word on a linguistic processing dimension, resulted in a much higher 
level of target identification. From this it may be reasonably assumed that the increased performance was brought about by the new mask's contributing to the processes needed for accessing the target's lexical representation (Lukatela \& Turvey, 1990a; Lukatela, Turvey, \& Todorović, 1991; Perfetti \& Bell, 1991; Perfetti et al., 1988).

In light of the foregoing assumptions, phonological information activated during the incomplete identification process for a masked word should be more enhanced by a letter string mask that is phonologically similar to the target word than by an otherwise similar letter string that is phonologically dissimilar to the target word (Lukatela \& Turvey, 1990a; Perfetti \& Bell, 1991; Perfetti et al., 1988). If a phonologically similar mask reinstates already activated phonological information of the target word, and if the semantic category decision task is sensitive to the early availability of phonological information prior to identification, then false positives should increase under backward masking by phonologically similar stimuli.

Empirical verification of this prediction is likely to be difficult. Demonstrating the effects of target homophony in the semantic categorization task and of mask phonological similarity in the brief exposure-linguistic backward masking task depends on achieving conditions of presentation that restrict the dependent measure to highly circumscribed ranges. In semantic categorization, relatively brief exposures must be coupled with instructions to respond rapidly. With time to ponder, subjects would check the homophone's orthography more carefully and detect the mismatch between the homophone and the lexical item that does belong to the designated category. In the backward linguistic masking task, parameters of stimulus presentation must be selected so that (1) the word target is effectively masked by the pseudoword mask qua visual pattern, but not so thoroughly as to prohibit influences of the pseudoword mask qua linguistic structure; and (2) the pseudoword mask is effectively masked by the subsequent pattern mask to the point that explicit recognition of its linguistic structure is ruled out, but its ability to hinder target processing is left intact. The presentation subtleties and theoretically unusual outcomes of "three-field" masking experimental procedures have been documented (Michaels \& Turvey, 1979; Turvey, 1978; Uttal, 1969). It is important to recognize that the two procedures involve very different performance baselines. In the Van Orden procedure, errors in semantic categorization are of the order of $10 \%$ (see the unmasked condition of the present Experiment 1). In contrast, the backward linguistic masking effects in the Perfetti procedure involve error rates in word identification in excess of $40 \%$. Consequently, the juxtaposition of the two procedures must necessarily entail errors in semantic categorization that are proportionally much greater than those ordinarily observed in the pure semantic categorization task.

The challenges of successfully juxtaposing the Van Orden and Perfetti procedures encourage a conservative stance toward the expected outcomes of the present experiment. Consider the target WEAK followed by "feek" (a phonologically similar pseudoword mask in lowercase), "felk" (a graphemic control for the homophonic mask in lowercase), and "furt" (a phonologically and graphemically dissimilar control mask in lowercase). The outcome most strongly favorable to the hypothesis that phonological codes are early sources of constraint on word processing would be more false recognitions of WEAK as A UNIT OF TIME in the masking condition WEAK-feek, than in either of the masking conditions WEAK-felk or WEAKfurt. A less strongly favorable outcome, but one that is consistent with the hypothesis, would be a larger difference between the WEAK-feek and WEAK-furt conditions than between the WEAK-felk and WEAK-furt conditions.

The conservatism encouraged by the juxtaposing of the two experimental procedures is reinforced by the contrast in the critical masking stimuli. The phonologically similar mask (feek) and its graphemic control (felk) are distinguished by a single phoneme. There are reasons for expecting similar rates of false positives under masking by felk and masking by feek. Following arguments by Perfetti and Bell (1991), the graphemic control mask felk would reinstate the abstract graphemic information already activated by WEAK. Thus, any tendency to respond to WEAK as A UNIT OF TIME under the control mask furt should be exaggerated under felk, because felk would make available more information about WEAK than would be made available under furk. With respect to the phonologically similar mask feek, it would reinstate both graphemic and phonological information-that is, it would reinstate more information about WEAK than felk. Hence, any tendency to respond to WEAK as A UNIT OF TIME under the control mask furt would be even more exaggerated under feek, because feek would make available even more information about WEAK. On these considerations, there should be a difference in false positives between WEAKfeek and WEAK-felk, but the difference need not be large. In consequence, confirmation of the early phonological constraint hypothesis seems more likely to be found in answering the following question: How different are WEAK-feek and WEAK-felk from the baseline provided by WEAK-furt?

\section{Method}

Subjects. Seventy-one undergraduate students at the University of Connecticut participated in the experiment. All were native English speakers, and had normal or corrected-to-normal vision. The subjects received credit for a course requirement.

Materials. A single trial consisted of a sequence containing a target word, a pseudoword mask, and a pattern mask, in that order. The target was always printed in uppercase and the pseudoword mask was always printed in lowercase. (This relation between stimulus type and case is the opposite of that used by Perfetti and colleagues [Perfetti \& Bell, 1991; Perfetti et al., 1988]; in their experiments, the target was the lowercase stimulus. The reason for the reversal was simply that the target stimuli of the present experiment were from the set of stimuli used in Experiment 1, which had all been presented in uppercase.) There were three conditions distinguished by the type of pseudoword mask: A given target was followed by a phonologically similar pseudoword (WEAK-feek), a graphemic control for the phonologically similar pseudoword (WEAK-felk), or an unrelated pseudoword (WEAK-furt). The target and the pseudoword mask always differed in the initial letter. 
The pattern mask consisted of pieces of letter shapes overlaid by a random dot pattern. The target, the pseudoword mask, and the pattern mask were equated in size both horizontally and vertically. (To equate the sizes of stimuli such as WEAK and feek, the uppercase letters were in 12 point and the lowercase letters were in 14 point.) Half of the targets were homophonic to the category exemplar (e.g., WEAK for the category A UNIT OF TIME). The remaining half of the targets were category exemplars (e.g., HOUR for the category A UNIT OF TIME). The original target stimuli from Van Orden (1987) were used in the experiment. Appendix B lists the critical target stimuli and those homophonic to the category exemplars, together with their pseudoword masks.

Procedure. The subjects sat at a Scientific Prototype six-channel tachistoscope with their eyes focused on a binocular fixation point. On each trial, a subject was first presented with a category name read aloud by the experimenter which was immediately followed by the presentation of a trial sequence. The target word was presented to the left eye for $40 \mathrm{msec}$, followed at an ISI of $5 \mathrm{msec}$ by the pseudoword mask presented to the right eye for $30 \mathrm{msec}$, thus producing dichoptic masking. Parametric studies have shown that these conditions occur at the end of central integrative processes and at the beginning of central interference processes (Michaels \& Turvey, 1979). The pseudoword mask was immediately followed (ISI $=\mathbf{0} \mathrm{msec}$ ) by the pattern mask on the same eye for $40 \mathrm{msec}$, monoptically masking the pseudoword mask. Again, parametric studies have shown that these conditions occur at the end of peripheral integrative processes and at the beginning of central interference processes (Michaels \& Turvey, 1979). The particular importance of the latter monoptic masking is that it severely delimits the time available for explicitly recognizing the pseudoword mask, thereby rendering an explicit appreciation of the connection between the target word and the pseudoword unlikely on those trials in which such a relation holds (e.g., WEAK-feek). The subject was instructed to respond orally with "yes" if the target word was the category exemplar and with "no" if it was not. As in Experiment 1, both speed and accuracy were stressed.

On half of the experimental trials the targets were homophones. Each subject saw a target word three times-once followed by a phonologically similar pseudoword, once by a graphemically similar pseudoword, and once by an unrelated pseudoword. The set of 18 homophonic targets was divided at random into three subsets of six words each. There were three blocks of experimental trials. In each block, a given subset of targets was followed by one type of pseudoword mask. Consider the three targets WEAK, MAUL, and MEET. If WEAK occurred in Block 1 and was followed by a phonologically similar mask (feek), then it was followed in Block 2 by a graphemic mask (felk), and in Block 3 by an unrelated mask (furt). If MAUL, on the other hand, occurred in Block 1 and was followed by a graphemic mask (lail), then it was followed in Block 2 by an unrelated mask (pune), and in Block 3 by a phonologically similar mask (lawl). Likewise, if MEET was followed in Block 1 by an unrelated mask (falp), then it was followed in Block 2 by a phonologically similar mask (keat), and in Block 3 by a graphemic mask (kest). Thus, a given type of mask occurred equally often in each block, and a given target was always followed by a different type of mask, depending on the experimental block. This was true for both the targets that were homophonic to the category exemplar and the category exemplar targets. Using the same target under the three different masking conditions as a unit of comparison controls for the frequency of the category exemplars, the visual similarity of the target and the exemplar, and the nature of the category names (see Jared \& Seidenberg, 1991). Although the explicit manipulation of these factors may provide additional information about the behavior of the word recognition system (see below), the purpose of the present experiment was to demonstrate that the phonological masking effect of the Perfetti procedure is continuous with the homophonic effect of the Van Orden procedure.
Each subject received 18 practice trials that were immediately followed by 108 experimental trials. At the beginning of the practice trials, the viewing parameters were set so that each subject could see the target word easily. On every third trial, the exposure duration of the target word was decreased by $10 \mathrm{msec}$. For the last three practice trials, the duration of the target was brought down to $40 \mathrm{msec}$. This duration was kept throughout the experiment. All the other parameters were kept constant from the beginning of the practice session.

\section{Results and Discussion}

Only the error data from the homophonic targets (e.g., WEAK) were analyzed. The data are summarized in Table 2. A one-way within-subject ANOVA was conducted on the percentage of false positives with three levels of type of pseudoword mask. The main effect of mask type (feek $=53 \%$ vs. felk $=48 \%$ vs. furt $=41 \%$ ) was significant for both subjects and stimuli $[F(2,140)=22.14$, $p<.001$, and $F(2,34)=4.82, p<.05$, respectively] as well as under the combined analysis [minimum $F^{\prime}(2,50)$ $=4.75, p<.05]$. Given the nature of the experimental procedure, the results warrant the conclusion that false positives were affected by mask type and also warrant the planned comparisons. With respect to the ideal hypothesized outcome, the $5 \%$ difference between WEAK-feek and WEAK-felk was not significant [minimum $F^{\prime}(1,50)=$ $1.73, p>.05$; for subjects, $F(1,140)=9.91, p<.005$; for stimuli, $F(1,34)=2.1, p>.05]$. Confirmation was found, however, for the next best hypothesized outcome. WEAK-feek (phonological masking) produced significantly more false positives than WEAK-furt [unrelated control masking, $53 \%$ vs, $41 \%$, minimum $F^{\prime}(1,50)=7.9$, $p<.01$; for subjects, $F(1,140)=44.24, p<.001$; for stimuli, $F(1,34)=9.63, p<.005]$. In contrast, the false positives for WEAK-felk (graphemic control masking) were not significantly different from those for WEAK-furt [48\% vs. $41 \%$, minimum $F^{\prime}(1,50)=2.24, p>.05$; for subjects, $F(1,140)=12.27, p<.001$; for stimuli, $F(1,34)$ $=2.74, p>.05]$. Because two of the graphemic controls had fewer matching letters to the target compared with the phonologically similar mask, these two targets were eliminated and an additional analysis was performed on the remaining 16 targets. The main effect of mask type (feek $=53 \%$ vs. felk $=48 \%$ vs. furt $=40 \%$ ) remained significant for both subjects and stimuli $[F(2,140)=$ $25.89, p<.001$, and $F(2,30)=5.81, p<.01$, respectively] as well as under the combined analysis [minimum $\left.F^{\prime}(2,44)=4.75, p<.05\right]$. The difference between WEAK-feek and WEAK-felk remained insignificant [minimum $F^{\prime}(1,44)=1.61, p>.05$; for subjects, $F(1,140)$

Table 2

Mean Percentages of False-Positive Responses and Standard Deviations in Experiment 2

Type of Mask

\begin{tabular}{|c|c|c|c|c|c|}
\hline \multicolumn{2}{|c|}{ Phonological } & \multicolumn{2}{|c|}{ Graphemic } & \multicolumn{2}{|c|}{ Unrelated } \\
\hline$M$ & $S D$ & $M$ & $S D$ & $M$ & $S D$ \\
\hline 53 & 13.39 & 48 & 10.30 & 41 & 10.05 \\
\hline
\end{tabular}


$=8.79, p<.005$; for stimuli, $F(1,30)=1.97, p>$ $.05]$. WEAK-feek again produced significantly more false positives than WEAK-furt [ $53 \%$ vs. $40 \%$, minimum $F^{\prime}(1,44)$ $=9.4, p<.01$; for subjects, $F(1,140)=51.23, p<$ .001 ; for stimuli, $F(1,30)=11.51, p<.005]$. The false positives for WEAK-felk (graphemic control masking) remained not significantly different from those for WEAKfurt $\left[48 \%\right.$ vs. $40 \%$, minimum $F^{\prime}(1,44)=3.23, p>.05$; for subjects, $F(1,140)=17.60, p<.001$; for stimuli, $F(1,30)=3.95, p>.05]$.

The categories of the present experiment were divided into those that have relatively few members (e.g., A SPHERICAL OBJECT) and those that have relatively many members (e.g., A WILD ANIMAL). It has been suggested that the less narrow the category, the more frequency dependent the homophonic effect becomes in semantic categorization (Jared \& Seidenberg, 1991). The potential significance of a frequency-dependent effect is that it implicates the involvement of a lexical (addressed phonology) route rather than a nonlexical (assembled phonology) route. A check was conducted on the possible frequency dependency of the WEAK-felk versus WEAK-furt contrast. The correlation of the contrast with word frequency was insignificant $(p>.05)$. Additionally, using the Kučera and Francis (1967) norms, the targets were divided into high-frequency targets (mean word frequency $=100.4$ ) and low-frequency targets (mean word frequency $=7.8$ ). Subsequently, a two-way ANOVA with factors of mask type and frequency (high-frequency vs. low-frequency targets) was performed on the false positives. The difference between high-frequency and low-frequency targets $(50 \%$ vs. $45 \%)$ was not significant under the combined analysis [minimum $F^{\prime}(1,19)<1$; for subjects, $F(1,70)=$ $10.14, p<.01$; for stimuli, $F(1,16)<1$ ]. As before, the main effect of mask type was significant for both subjects $(p<.001)$ and stimuli $(p<.05)$ as well as under the combined analysis $(p<.05)$. The phonological mask produced significantly more errors when compared with the unrelated-mask condition for high-frequency targets [56\% vs. $44 \%$; for subjects, $F(1,70)=25.37, p<.001$; for stimuli, $F(1,8)=5.00, p<.05]$ as well as for lowfrequency targets [50\% vs. $39 \%$; for subjects, $F(1,70)=$ $17.45, p<.001$; for stimuli, $F(1,8)=4.08, p=.06$ ] The frequency $\times$ mask type interaction was not significant for either subjects or items (both $F$ s $<1$ ). An additional analysis, performed on the set of data after the two questionable targets were removed, replicated the preceding pattern of significant and insignificant effects with the exception of a significant $(p<.05)$ stimuli analysis for low-frequency items in the contrast between phonological and unrelated masks.

To test the assumption that category size affects the frequency dependence of the homophonic effect in semantic categorization, an additional three-way ANOVA was conducted with factors of mask type (phonological, graphemic, unrelated), frequency (high or low), and category size (many or few). Category size proved insignificant [minimum $F^{\prime}(1,17)<1$; for subjects, $F(1,70)=10.49$, $p<.005$; for stimuli, $F(1,14)<1$ ]. None of the two- way interactions (mask type $x$ frequency, mask type $x$ category size, frequency $\times$ category size) were significant in either the subject or stimulus analysis. The three-way interaction was not significant in either of the analyses (both $F \mathrm{~s}<1$ ). The same pattern of significant and insignificant effects was replicated in the additional analysis after the two questionable targets were removed.

The consistency of the elevated percentage of errors under the phonological mask in comparison with the unrelated mask agrees with previous results indicating the benefits of phonological masking (e.g., Perfetti \& Bell, 1991). At the same time, the absence of frequency and category size effects and the lack of interactions with the homophonic effect disagree with recent results highlighting the importance of the category size variable (Jared \& Seidenberg, 1991). In part, the failure to confirm a dependency of the homophonic effect on category size may be due to the diversity of the category names used by Jared and Seidenberg. The extensions of their category names were very large (e.g., A LIVING THING, AN OBJECT). In the present experiment, the extensions of the "broad" category names were large, but not extremely so (e.g., A WILD ANIMAL, A TYPE OF FOOD). It could be the case that relatively unlimited category designations such as A LIVING THING are inappropriate contexts for demonstrating homophonic effects because they present too many illdefined processing options. Jared and Seidenberg examined category labels that were at the opposite extreme from those used in 1987 by Van Orden (e.g., A MEMBER OF A CONVENT). In the present experiment, intermediate category labels were examined.

In the present experiment, we used a particular version of three-field masking (Michaels \& Turvey, 1979; Turvey, 1978) that was originally suggested by Naish (1980) and further developed by Perfetti (Perfetti \& Bell, 1991; Perfetti et al., 1988) to investigate the time course of codes in visual word recognition. The three fields were constituted by stimuli such as the WEAK-feek pattern mask presented as right-eye stimulus/left-eye stimulus/left-eye stimulus. For the present purposes, the major benefit of the three-field masking procedure with dichoptic and monoptic presentation is that it provides a sensitive means for controlling the time available for processing both the primary (e.g., WEAK) and the secondary (e.g., feek) stimuli. Any changes in the perception of the primary stimulus as a function of the secondary stimulus must be attributable to (1) codes activated rapidly by both stimuli, and (2) dimensions of the secondary stimulus's codes that have a particular bearing on the processing of the primary stimulus.

The principal question of interest in the present study was whether or not the phonological relation between a pseudoword mask and its target could influence falsepositive responding in semantic categorization. If it could, then there would be further support for the argument that phonological codes are early sources of constraint on word identification. Respecting the challenges posed by the subtleties of the two tasks integrated in the present experiment, it seems reasonable to conclude from the re- 
sults that false-positive responses (to target words that are homophonic with words belonging to the designated categories) were systematically affected by the nature of the pseudoword mask. In particular, the results were consistent with the expectation that more false positives should be engendered under phonologically similar pseudoword masks. If it is correct to assume that a phonologically similar backward mask reinstates phonological information activated during the incomplete identification of the target (Lukatela \& Turvey, 1990a; Perfetti \& Bell, 1991; Perfetti et al., 1988), then it would have to be concluded from the results of Experiment 2 that semantic categorization involves phonological information at a very early stage. Moreover, the results suggest that the effects of early phonological activation on accessing a word's meaning are not restricted to only low-frequency words, but hold for high-frequency words as well.

\section{GENERAL DISCUSSION}

An important expectation from a number of accounts of the process of visual word recognition is that the constraints imposed by phonological codes occur only in its later stages (see Van Orden et al., 1990, for a review). Overall, the results of the present two experiments are not very supportive of this delayed-phonology hypothesis, but are more suggestive of a rapid, early contribution of phonology to word recognition. In particular, the outcome of Experiment 2 suggests that phonological codes are activated before a word's lexical representation is accessed.

The results of the present two experiments are favorable to the phonological coherence hypothesis advanced by Van Orden et al. (1990), which is based on the understanding that the covariation between orthographic and phonological structure more closely approximates a singlevalued map than the covariation between orthographic structure and other linguistic structures (e.g., semantic or syntactic). The consequence of single valuedness in dynamic terms is that resonance or self-consistency is achieved rapidly within the connective matrix linking the processing units of the two structures. As expressed in adaptive resonance theory (Grossberg, 1987; Grossberg $\&$ Stone, 1986), a resonant mode is achieved when the activity excited in a given layer of processing units from below matches that excited from above. ${ }^{1}$ Given this, the phonological coherence hypothesis amounts to the claim that phonological codes are coded at the outset closest to their respective attractors and, therefore, are the earliest to achieve resonance or coherence (Van Orden et al., 1990). They can, thereby, act to stabilize other concurrent and ongoing linguistic codings. By this hypothesis, the persistence of the homophonic effects under the pattern masking of Experiment 1, and their amplification under the homophonous masking of Experiment 2, occurred because phonological codes cohere rapidly and provide, thereby, immediately available constraints on processing.

\section{REFERENCES}

Coltheart, M. (1978). Lexical access in simple reading tasks. In G. Underwood (Ed.), Strategies of information processing (pp. 151216). London: Academic Press.

Coltheart, M., Davelaar, E., Jonasson, J. T., \& Besner, D. (1977). Access to the internal lexicon. In S. Dornic (Ed.), Attention and performance VI (pp. 535-555). New York: Academic Press.

GrossberG, S. (1987). Competitive learning: From interactive activation to adaptive resonance. Cognitive Science, 11, 23-63.

Grossberg, S. , \& STONE, G. O. (1986). Neural dynamics of word recognition and recall: Priming, learning, and resonance. Psychological Review, 93, 46-74.

Humphreys, G. W., \& Evett, L. J. (1985). Are there independent lexical and nonlexical routes in word processing? An evaluation of the dual-route theory of reading. Behavioral \& Brain Sciences, 8, 689-740.

JARED, D., \& SEIDENBERG, M. S. (1991). Does word identification proceed from spelling to sound to meaning? Journal of Experimental Psychology: General, 120, 358-394.

KuCERA, H., \& Francis, W. N. (1967). Computational analysis of present-day American English. Providence, RI: Brown University Press.

Lukatela, G., \& Turvey, M. T. (1990a). Automatic and prelexical computation of phonology in visual word identification. European Journal of Cognitive Psychology, 2, 325-343.

Lukatela, G., \& Turvey, M. T. (1990b). Phonemic similarity effects and prelexical phonology. Memory \& Cognition, 18, 128-152.

Lukatela, G., \& Turvey, M. T. (1991). Phonological access of the lexicon: Evidence from associative priming with pseudohomophones. Journal of Experimental Psychology: Human Perception \& Performance, 17, 951-966.

LUkATEla, G., \& TuRvey, M. T. (1993). Similar attentional, frequency, and associative effects for pseudohomophones and words. Journal of Experimental Psychology: Human Perception \& Peformance, 19, 166-178.

Lukatela, G., Turvey, M. T., \& Todorović, D. (1991). Is alphabet biasing in bialphabetical word perception automatic and prelexical? Journal of Experimental Psychology: Learning, Memory, \& Cognition, 17, 653-663.

MCCusker, L. X., Hillinger, M. L., \& Bias, R. G. (1981). Phonological recoding and reading. Psychological Bulletin, 89, 217-245.

Michaels, C. F., \& Turvey, M. T. (1979). Central sources of visual masking: Indexing the structures supporting seeing at a single brief glance. Psychological Research, 41, 1-61.

NAISH, P. (1980). The effects of graphemic and phonemic similarity between targets and masks in a backward visual masking paradigm. Quarterly Journal of Experimental Psychology, 32, 57-68.

Perfetti, C. A., \& Bell, L. C. (1991). Phoneme activation during the first $40 \mathrm{msec}$ of word identification: Evidence from backward masking and priming. Journal of Memory \& Language, 30, 473-485.

Perfetti, C. A., Bell, L. C., \& Delaney, S. (1988). Automatic (prelexical) phonetic activation in silent word reading: Evidence from backward masking. Journal of Memory \& Language, 27, 59-70.

Perfetti, C. A., Zang, S., \& Berent, I. (1992). Reading in English and Chinese: Evidence for a "universal" phonological principle. In R. Frost \& L. Katz (Eds.), Orthography, phonology, morphology, and meaning (pp. 227-248). Amsterdam: North-Holland.

SEIDENBERG, M. S. (1985). The time course of phonological code activation in two writing systems. Cognition, 19, 1-30.

TURVEY, M. T. (1973). On peripheral and central processes in vision: Inferences from an information-processing analysis of masking with patterned stimuli. Psychological Review, 80, 1-52.

TURVEY, M. T. (1978). Visual processing and short term memory. In W. K. Estes (Ed.), Handbook of learning and cognitive process $V$ (pp. 91-140). Hillsdale, NJ: Erlbaum.

UTTAL, W. R. (1969). The character in the hole experiment: Interaction of forward and backward masking of alphabetic character recognition by dynamic visual noise (DVN). Perception \& Psychophysics, 6. $177-181$. 
VAN Orden, G. C. (1987). A rows is a ROSE: Spelling, sound, and reading. Memory \& Cognition, 15, 181-198.

Van Orden, G. C., Johnston, J. C., \& Hale, B. L. (1988). Word identification in reading proceeds from spelling to sound to meaning. Journal of Experimental Psychology: Learning, Memory, \& Cognition, 14, 371-385.

Van Orden, G. C., Pennington, B. F., \& Stone, G. O. (1990). Word identification in reading and the promise of subsymbolic psycholinguistics. Psychological Review, 97, 488-522.
NOTE

1. It is important to note that the interpretation that backward masking curtails processing, which was developed under the auspices of classical models of information processing, is essentially adhered to in adaptive resonance theory. Within this contemporary form of informationprocessing theory, a visual pattern mask prohibits resonance sharpening and equilibration through the exclusion of top-down templatematching signals (Grossberg \& Stone, 1986, pp. 62-63).

\section{APPENDIX A}

Category Names, Homophonic Targets, and Graphemic Controls in Experiment 1

\begin{tabular}{lll}
\hline \multicolumn{1}{c}{ Category } & Homophonic Target & Graphemic Control \\
\hline A feature of a person's abdomen & NAVAL & NOVEL \\
A deer & DOUGH & DOUBT \\
A type of hotel room & SWEET & SHEET \\
A member of a convent & NONE & NINE \\
A small stream & CREAK & CHEEK \\
A flower & ROWS & ROBS \\
A part of a dress & SEEM & SLAM \\
A wild animal & BORE & BORN \\
A kitchen utensil & BOLL & BOIL \\
A part of a mountain & PEEK & PECK \\
A feature of an ocean shore & BEECH & BENCH \\
A part of a person's face & KNOWS & SNOBS \\
Something caused by gravity & WAIT & WRT \\
Organized group of people & TEEM & TERM \\
A servant & MADE & MAIN \\
A part of a horse's bridle & RAIN & RUIN \\
A biblical religious leader & PROFIT & PROTEST \\
A type of food & MEET & MELT \\
A breakfast food & SERIAL & VERBAL \\
An ancient musical instrument & LOOT & LOST \\
\hline
\end{tabular}

\section{APPENDIX B}

Category Names, Homophonic Targets, Phonologically Similar Masks, Graphemic Masks, and Unrelated Masks in Experiment 2

\begin{tabular}{lllll}
\hline & & \multicolumn{3}{c}{ Mask } \\
\cline { 3 - 5 } \multicolumn{1}{c}{ Category } & Target & Phonological & Graphemic & Unrelated \\
\hline A place to shop & MAUL & lawl & lail & pune \\
A part of a human body & FEAT & reet & remt & wolk \\
A part of a horse's body & MAIN & fane & farn & neep \\
A vegetable & BEAT & leet & lert & flom \\
A part of a house & HAUL & vawl & varl & shat \\
A bird & TURN & gern & geen & feep \\
An edible plant & LEAK & zeek & zenk & kive \\
A part of a human body & HARE & jair & jawr & gube \\
A group of people & TEEM & neam & nerm & scok \\
A type of food & MEET & keat & kest & falp \\
A kitchen utensil & BOLL & doal & doil & dups \\
A part of a woman's dress & SEEM & jeam & jelm & tlay \\
A spherical object & BAWL & vole & vols & kint \\
A wild animal & BORE & gawr & gair & jurp \\
A servant & MADE & kayd & kald & pibe \\
A part of a mountain & PEEK & heak & helk & wape \\
A unit of time & WEAK & feek & felk & furt \\
A person of medieval time & SURF & merf & meif & tilk \\
\hline
\end{tabular}

